\title{
DEVELOPMENT OF BASE TRANSCEIVER STATION SELECTION ALGORITHM FOR COLLOCATION ARRANGEMENT
}

\author{
T. M. Ajibola ${ }^{1}$, N. T. Surajudeen-Bakinde ${ }^{2,{ }^{*}}$ and S. A. Y. Amuda ${ }^{3}$

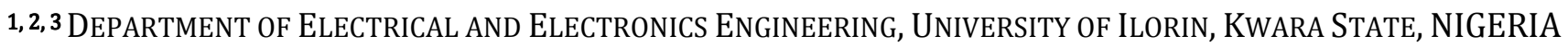 \\ E-mail addresses: ${ }^{1}$ taiye.ajibola@yahoo.com, ${ }^{2}$ deenmat1211@gmail.com, ${ }^{3}$ amudasulyman@gmail.com
}

\begin{abstract}
Placement of base transceiver station (BTSs) by different operators on a particular site as collocation site, so as to save cost and reduce the number of people who are at risk of radiation in BTSs located places as compared to each operator having different BTSs is the new trend in Nigeria telecommunication industries development. Collocation of base stations is much safer when compared to scattered base station telecommunication operators because of the radiations. Although the International Commission on Non-Ionizing Radiation Protection (ICNIRP) viewed that the presently existing scientific verification that prolonged contact with low frequency magnetic fields is more often than not connected with an increased danger of childhood leukaemia is too frail to form the basis for exposure guidelines. This work includes the study, analysis and proposal of a collocation scheme based on collected data of the number of BTS already sited in University of Ilorin, Ilorin, Nigeria as a case study. A drive test conducted with Transmission Environmental Monitoring System (TEMS) equipment was carried out on the existing BTSs, and a linear algorithm optimization program based on the spectral link efficiency of each BTS was developed, the output of this site optimization gives the selected number of base station sites to be used for the collocation arrangement, and the BTSS site with the best spectral link efficiency are selected in accordance with the output of the site optimization for the collocation.
\end{abstract}

Keywords: absolute radio frequency channel number; base transceiver station; collocation; radiation; spectral link efficiency.

\section{INTRODUCTION}

The deployment of many base transceiver stations (BTSs) by different telecommunication operators, scattered all over the vicinity of residence, work and schools, has become a great concern for economic reasons and exposure to radiation by the general public within the BTS environment.

A BTS is a permanent location in a mobile cellular network used for radio communications with mobile units. The major work of a BTS is to allow a good process of information transmission and reception between mobile stations [1]. The base station consists of radio channels each having its absolute radio frequency channel number (ARFCN), transmitter and receiver antennas. These antennas which are mostly directional antennas for mobile telecommunications are positioned strategically on a tower in order to render services to certain geographical area. The location of a BTS is of crucial importance; the positioning of the BTS as well as the strategic inclination of the communication antennas can influence the quality of service rendered to the custumers. Due to this reason some base stations are located on a hilly terrain free from blockage of any structures in order to enhance quality connectivity and coverage [1]. Each BTS has between 1-16 transceivers depending on the density of users in the cell [2].

The Federal Communications Commission (FCC) defines collocation as the mounting or installation of antennas from different GSM operators on an existing tower, building or structure for the purpose of transmitting and/or receiving radio frequency signals for communications purposes [2].

In 2001 when Global System for Mobile Communications (GSM) was introduced in Nigeria, Nigerians embraced it with a lot of passion to enjoy all the benefits of the new mobile communications 
technology. The pioneer then was Econet Wireless (now Airtel) and MTN before Globacom was licensed in 2003. Etisalat joined the race of telecoms service providers in 2008. Full scale service delivery was enjoyed as calls went through without the slightest congestion. As the number of subscribers increase, it was expected that telecoms operators will increase infrastructure rollout at a commensurate level to that of subscriber's growth. But little attention was paid to infrastructure improvement until a time when network congestion sets in, leading to subscribers experiencing poor services delivery [3]. Nigerian government policy had consistently put pressure on the GSM operators to improve their QoS with projection that there will be need for about 60,000 BTSs by 2018 [2] and one of the ways to achieve this target is to allow collocation scheme among telecommunication operators.

The health and cost implications of the increasing number of these BTSs influence both the government and the Association of Licensed Telecoms Operators of Nigeria (ALTON) to encourage collocation by proposing the appropriate regulations and legislations for the collocation scheme [4]. This collocation scheme is not only to reduce telecoms infrastructures but also to manage the system's energy and to ensure increase in broadband penetration and wider coverage area without cross-signal or noise interference.

Human health threat becomes the consequent issue of the increasing BTS sites as non - ionizing radiations from base station sites have been reported to cause considerable health hazards, such as brain tumor, eye cancer to mention but a few [5] even though these health effects have not been medically proven. The main fear is focused on long-term exposure to these radiations. This concern especially originated from the use of some mobile phones and human closeness to base stations for a long time, as some studies have shown that the radiations from these two sources were associated with some health problems like birth defects, brain tumors, lymphomas, and memory problems, [5]

The first developmental step was taken by the Nigerian Communication Commission (NCC) by issuing the Guidelines on Collocation and Infrastructure Sharing with presumption that it will reduce or eliminate avoidable costs in the telecommunications industry, including capital expenditure on supporting infrastructure [6]. The guidelines established a framework within which operators can negotiate collocation and infrastructure-sharing arrangements, as well as promoting fair competition through the grant of equal access to operators' installations and facilities on mutually agreed terms. Rule 4 of the guidelines specifies the infrastructure to be shared, as encouraged by the NCC as itemized thus:

- $\quad$ Rights of way

- $\quad$ Masts, poles, antennae and tower structures

- $\quad$ Ducts, trenches and spaces in buildings

- $\quad$ Electricity (from public or private sources)

[6].

Where the sharing of infrastructure such as rights of way and electricity is conditional on securing approval from a granting authority, such approval should be obtained before the sharing arrangement can be finalised [6].

\section{RELATED WORKS}

The principal mandate of NCC as stated in its guideline for collocation is to launch a structure within which operators can discuss collocation arrangements, and check that the occurrence of avoidable duplication of infrastructure is minimized or totally avoided and to keep the environment by reducing the spread of infrastructure and facilities installation, [7].

The collocation arrangement definitely cause physical significant changes to existing wireless mast or base station. The significant change can be in the form of placement of a proposed antenna that would involve adding an extension to the body of the mast to protect the antenna from severe weather or to connect the antenna to the mast through cable. Currently the most commonly shared infrastructure among operators is passive infrastructure, as it is easier to contract its setup and maintenance, [8].

Ever since the introduction of telecommunication, the equipment and the deployment of the infrastructure of whatever type has been resource demanding from a business point of view, operators in the recent past have embraced collocation of base station masts. The cost of sharing facilities in a collocation arrangement is practically low, compared to the cost of constructing one's own infrastructure, for this reason there is faster returns on investment and an opportunity to focus more on the core business of the companies by providing telecoms services [8].

The challenges facing the optimal selection of base station transceiver site for collocation are the non uniformity in the quality of service in a geographical location, coverage area and the interference of neighboring base station sites. The challenge of 
optimum selection of BTSs was considered using the simulated annealing optimization method, as in [9], illustrated that credibly BTSs sites can be successfully selected not considering how unplanned the starting position of the BTSs were at the initial state of the selection. The factors considered to achieve this simulated annealing optimization model include, the path loss with line-of-sight constructed as intermediate between the upper bound and the lower bound, the height of the mobile station antenna as well as the base station antenna were also put into the consideration in order to achieve this result [9].

Another method used for BTSs selection is the handover method. Handover algorithm using fuzzy logic optimization method was done in [10]. The method was used to select a base station based on the received signal strength, distances from neighboring base station, bandwidth usage and the velocity of the mobile equipment. The simulation is based on Sugeno reasoning method, as it works well with optimization and adaptive techniques [10].

The Majorization - Minimization algorithm was also used for base station selection for energy efficient network operation. The dynamic energy consumption of each of the base station was considered in order to reduce the energy consumed by these base stations and to determine the number of base stations that will remain active at a particular time while the others are shutdown in order to reduce energy consumption [11].

In this work, the factors considered for the base station selection and which were also used for the spectral link efficiency calculation include the absolute radio frequency channel number (ARFCN), the equivalent value for the mobile station power control level, carrier - to - interference and the frequency band of the carrier signal. The carrier frequency is assigned by the ARFCN. A fraction of the overall number of channels accessible to the entire system is assigned to each base station, and when base stations are in close proximity to each other they are assigned different group of channels so that the interference between base stations is minimized [11]. Though selection of base station using various techniques had been ongoing, this research work thereby pioneered the exploration of BTS selection based on Spectral link Efficiency using the GSM layout at the University of Ilorin and its immediate environs as the case study.

This paper is organized as follows: Section one is the introduction while Section two reviews work of other researchers in the same field of work. The data collection method employed in achieving the goal of the research is described in Section three. Section four describes the system model for the work. Analyses of the results obtained are carried out in Section five, while the conclusion is drawn in Section six.

\section{SYSTEM MODEL}

University of Ilorin is represented as a suburban environment because this environment is characterized by widestreet, buildings with height generally less than three stories making diffractions over roof tops possible [12] and luckily with the four Nigerian GSM operators fully operational within the University. Base station deployment is represented as $M$ where $M=\left\{m_{1}, m_{2}, m_{3, \ldots . .} m_{k}\right\}$ with sets of mobile users $N$ where $N=\left\{n_{1}, n_{2}, n_{3, \ldots . . .} n_{z}\right\}$.

In order to achieve the model there is need to determine certain parameters, such as the spectral link efficiency, horsepower of a user and the bit rate.

The spectral link efficiency is the information rate (bits/s) that can be transmitted over a given frequency bandwidth $(\mathrm{Hz})$ in a specific communication system, which is the measure of network performance expressed in matrix form as in equation (1) below [13],

$$
w=\left[\begin{array}{cccc}
w_{11} & w_{12} & \ldots \ldots & w_{1 z} \\
w_{21} & w_{22} & \ldots \ldots & w_{2 z} \\
: & : & : & : \\
w_{k 1} & w_{k 2} & \ldots \ldots & w_{k z}
\end{array}\right]
$$

where $\omega_{k z}$ is the spectral link efficiency of base station site $k$ to a mobile user $z$ measured in $[\mathrm{bit} / \mathrm{s}] / \mathrm{Hz}$, and it is mathematically expressed as:

$$
w=\gamma_{k z}(B W) \log \left[1+\left[P_{k z} / \mu_{k z}(\text { SINR }) \sum_{d=1} P_{d z}+P_{z}(\text { Noise })\right]\right]
$$

where, $P_{z}$ (Noise) is the noise power of user $\mathrm{z}$ in $\mathrm{dBm}$. This was a calculated value from the received power (RxLev), $P_{k z}$ obtained from the conducted drive test and then substituted into equation (3).

$$
P_{z}(\text { Noise })=\frac{P_{k z}}{\log _{10}\left(\frac{C}{I}\right)}(d b m)
$$

where $\frac{C}{I}$ is the carrier to interference ratio

$\gamma_{k z}(B W)$ represents the suitable scaling factor known as bandwidth efficiency which is calculated as;

$$
\gamma_{k z}(B W)=\frac{f_{u}}{s_{t}} \times 100 \%
$$


where $f_{u}$ is the frequency in use and $s_{t}$ is the total allocated spectrum for the GSM band in use.

The frequency in use for GSM 1800 is expressed as 0.2 $\mathrm{x}$ [n (ARFCN)-512], while for GSM 900 it is expressed as $0.2 \mathrm{x}$ [ $\mathrm{n}(\mathrm{ARFCN})]$. Where $\mathrm{n}$ is the number of channels in the frequency.

$\mu_{k z}($ SINR $)$ represents the suitable scaling factor known as signal -to - interference -plus -noise ratio. The worst case signal-to-interference-plus-noise ratio is considered in order to take account of the inter-cell interference. It is calculated from the drive test result as:

$$
\mu_{k z}(\operatorname{SINR})=\frac{C / I(\text { full })}{C / I(s u b)}
$$

$P_{k z}$ represents the received signal power from base station $k$ to user $\mathrm{z}$ which is in $\mathrm{dBm}$ [10], this is obtained straight from the radio parameters displayed for the conducted drive test.

$P_{d z}$ is the dedicated power on the user $z$ mobile station [13]. From the TEMS status window under the radio parameters, it is displayed as mobile station power control level and this has a range of zero to thirty-one, each of these levels is decoded as power in dBm. [13]

Also All users have fixed QoS requirements represented by a minimum required data (bit) rate which is the rate at which bits are transferred from one location to another and it is expressed as:

$$
r=\left[\begin{array}{llll}
r_{1} & r_{2} & \ldots & r_{3}
\end{array}\right]
$$

In GSM (which is the area of concern in this research work), user's data rate is $9.6 \mathrm{kbps}$ per time slot [14] and in this paper this data rate is taken to be the same for all the mobile users in the telecommunication network.

Allocated bandwidth, to support this data rate, base station $k$ to which user $z$ is connected has to allocate bandwidth $b_{k z}$ given as;

$$
b_{k z}=\frac{r_{z}}{w_{k z}}
$$

where $\omega_{k z}$ is the spectral link efficiency and $r_{z}$ is the data rate of a mobile user $z$.

The allocated bandwidth can then be expressed in matrix form as

$$
\begin{aligned}
& X_{k z}=\left[\begin{array}{cccc}
x_{11} & x_{12} & \ldots . & x_{1 z} \\
x_{21} & x_{22} & \ldots & x_{2 z} \\
: & : & : & : \\
x_{k 1} & x_{k 2} & \ldots . & x_{k z}
\end{array}\right] \\
& X_{k z}=\left\{\begin{array}{l}
1 \\
0
\end{array} \equiv \in X_{i}(M x N)\right. \\
& \min _{X \in_{i}(M x N)}[w] \\
& P_{k z} x_{k z} \Rightarrow-90 \leq P_{k z} x_{k z} \leq-55 \\
& x_{k z} \Rightarrow 3 \leq b_{k z} x_{k z} \leq 47 \\
& x_{k z} \in\{k z\} k \in M, z \in N
\end{aligned}
$$

The state of network connection of a user, this is represented by matrix $\mathrm{X}$ which is,

$$
X_{k z}=\left\{\begin{array}{l}
1 \\
0
\end{array} \equiv X \in i(M x N)\right.
$$

Equation (9) is one if and only if $\mathrm{z}$ is connected to base station $\mathrm{k}$, otherwise, it is zero.

And can be represented in the matrix form as:

$$
X_{k z}=\left[\begin{array}{cccc}
x_{11} & x_{12} & \ldots & x_{1 z} \\
x_{21} & x_{22} & \ldots & x_{2 z} \\
: & : & : & : \\
x_{k 1} & x_{k 2} & \ldots & x_{k z}
\end{array}\right]
$$

When all these parameters are known, then the optimization problem can be solved. To select a base station based on the spectral link efficiency and thus reducing congestion of BTS sites in an area, then the following optimization problem is needed to be solved,

$$
\min _{X \in i}[w]
$$

subject to:

$P_{k z} x_{k z} \Rightarrow-90 \leq P_{k z} x_{k z} \leq-55 ; \quad x_{k z} \Rightarrow 3 \leq b_{k z} x_{k z} \leq 47$;

$x_{k z} \in\{k z\} k \in M, z \in N$

The first constraint forces every user to be connected to precisely one base station.

The second constraint makes certain that no user go beyond the bandwidth limit accessible to the base station.

Each row of $P$ and $B$ corresponds to a base station while each column corresponds to the mobile user and this refers to the four networks (MTN, GLO, AIRTEL and ETISALAT) operative within the campus.

\section{DATA COLLECTION METHODOLOGY}

In this paper, the method used is the collection of data (related to the model in equation (2) above) from some strategically designated units for monitoring the 
QoS of the four GSM operators in the area. A Global Positioning System (GPS) was used to know the location (longitude and latitude) of each of these base station sites and to monitor the speed limit of the drive. Also TEMS software was installed on a laptop, and connected to the USB port of the laptop was a dongle which serves as a license to the use of the software in order to carry out a drive test on the respective base station so as to know the base station radio parameters needed to achieve the model presented in Section 3.

The procedure used for the measurements is described from step one to step five as itemized thus:

1) Drive test was carried out on each of the existing telecommunication networks within the campus at an average speed of $30 \mathrm{~km} / \mathrm{hr}$ and this lasted for six minutes. Drive test was done along the main streets leading to the existing base station sites on the main campus.

2) The network performance of each of these base stations was observed from the drive test reading based on the radio parameters needed for the model equation in equation (2), these radio parameters include, received signal level (RxLev), carrier-to-interference (C/I), broadcast control channel absolute radio frequency channel number (BCCH ARFCN), mobile station power control level and the frequency (band) in use. The received signal level has a range of $-110 \mathrm{dBm}$ to $-30 \mathrm{dBm}$. The range of signal strength rated best is $-70 \mathrm{dBm}$ to $-30 \mathrm{dBm}$ while the poorest is between -110 $\mathrm{dBm}$ and $-90 \mathrm{dBm}$ [14].

3) The radio parameters stated in (2) were used to determine the noise power, bandwidth efficiency and the scaling factor for signal-to-interferenceplus-noise ratio as expressed in equations (3), (4) and (7) respectively.

4) The spectral link efficiency was determined by fixing the parameters obtained in (3) into equation (2).

5) An optimization program using linear algorithm was developed based on the spectral link efficiency through the TEMS to know the exact number of base station(s) to be selected for collocation arrangement.
Figure 1 shows the information window of TEMS during the drive test, each panel contains information required for the system model and are listed out in the steps of the procedure used for the measurement. The optimization technique used was linear algorithm.

\section{RESULTS AND DISCUSSION}

The results are presented in Tables 1, 2, 3 and 4 that will be discussed as follows. From Table 1, while using AIRTEL SIM card for Mobile Station 1, at AIRTEL base station the information transmitted over the allocated bandwidth of $3.31 \mathrm{kHz}$ was an average value of 2.90 ([bit/s]/Hz), while GLO base station has the highest transmitted information rate using the least allocated bandwidth, and this was followed by ETISALAT with spectral link efficiency of 2.98 ([bit/s]/Hz).

In Table 2, using MTN SIM card for Mobile Station 2, the received signal strength at AIRTEL base station was reduced from $-60 \mathrm{dBm}$ to $-72 \mathrm{dBm}$ and the information transmitted over the allocated bandwidth of $6.17 \mathrm{KHz}$ was 1.55 ([bit/s]/Hz) which was the highest value on this table, while this was followed by MTN base station at Stadium with spectral link efficiency of 1.53 ([bit/s]/Hz).

Table 1: Network performance of all base stations with respect to mobile user 1 (AIRTEL SIM)

\begin{tabular}{lccc}
\hline MOBILE STATION (USER) & $P_{k z}(\mathrm{dBm})$ & $\left.\omega_{k z}\left(\frac{b i t}{\mathrm{~Hz}}\right]\right)$ & $B_{k z}(\mathrm{KHz})$ \\
1(AIRTEL) & -60.00 & 2.90 & 3.31 \\
\hline BS 1 (AIRTEL-STADIUM) & -79.00 & 1.60 & 6.00 \\
BS 2 (MTN-CLINIC) & -55.00 & 2.55 & 3.76 \\
BS 3 (MTN-STADIUM) & -87.00 & 3.17 & 3.02 \\
BS 4 (GLO-LAW) & -95.00 & 2.98 & 3.23 \\
BS 5 (ETISALAT-LAW) & & & \\
\hline
\end{tabular}

Table 2: Network performance of all base stations with respect to mobile user 2 (MTN SIM)

\begin{tabular}{lccc}
\hline $\begin{array}{l}\text { MOBILE STATION 2 } \\
\text { (MTN) }\end{array}$ & $P_{i j}(\mathrm{dBm})$ & $\omega_{i j}\left(\frac{[\mathrm{bit}}{\mathrm{s}}\right]$ & $B_{i j}(\mathrm{KHz})$ \\
\hline $\begin{array}{l}\text { BS 1 (AIRTEL- } \\
\text { STADIUM) }\end{array}$ & -72.00 & 1.55 & 6.17 \\
BS 2 (MTN-CLINIC) & -71.00 & 1.38 & 6.97 \\
BS 3 (MTN-STADIUM) & -67.00 & 1.53 & 6.28 \\
BS 4 (GLO-LAW) & -81.00 & 1.51 & 6.36 \\
BS 5 (ETISALAT-LAW) & -87.00 & 1.15 & 8.33 \\
\hline
\end{tabular}




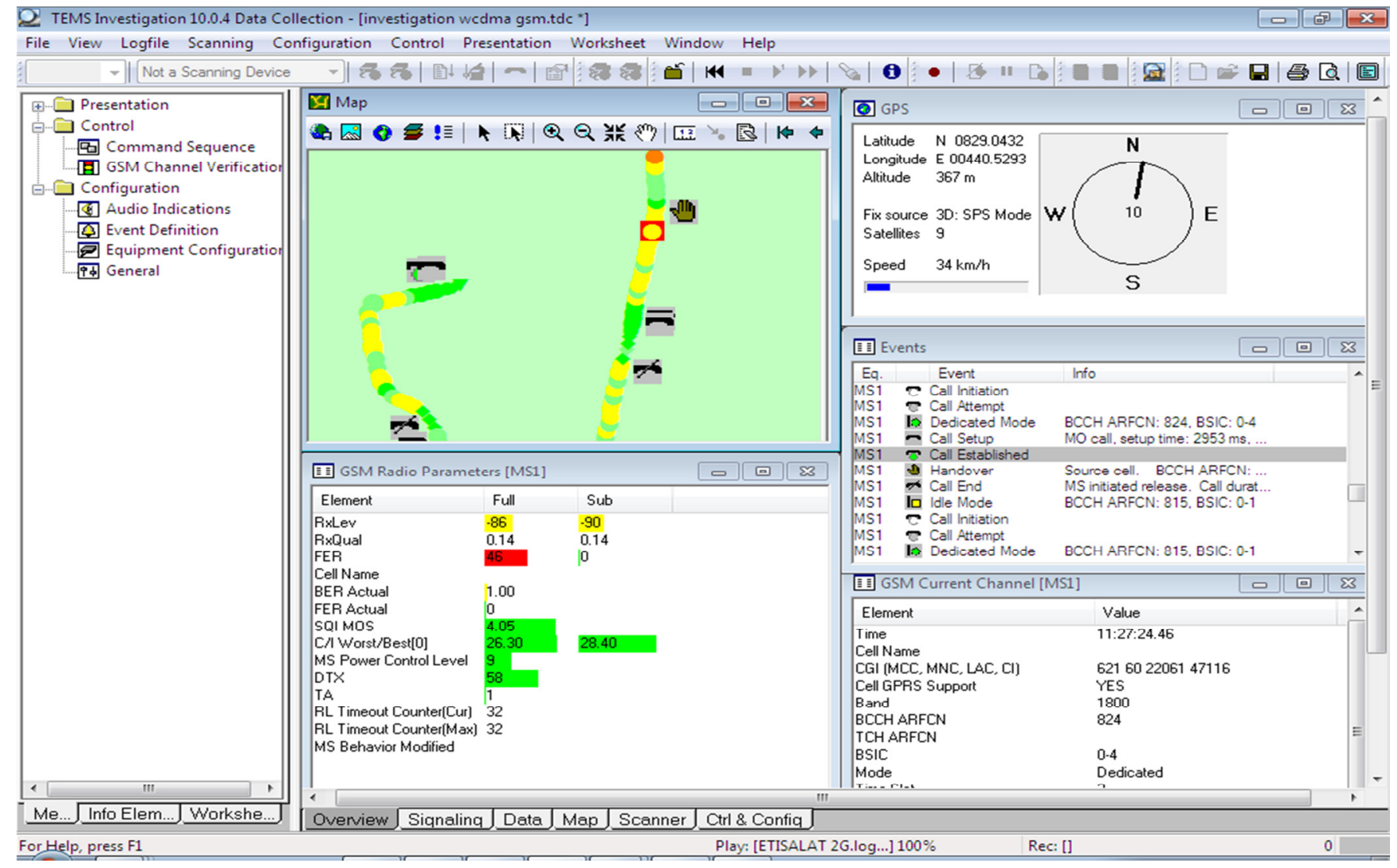

Figure 1: Screenshot of software used to display the radio parameters.

Table 3: Network performance of all base stations with respect to mobile user 3 (GLO SIM)

\begin{tabular}{|c|c|c|c|}
\hline $\begin{array}{c}\text { MOBILE STATION } 3 \\
\text { (GLO) }\end{array}$ & $P_{k z}(d B m)$ & $\omega_{k z}\left(\frac{\left[\frac{b i t}{s}\right]}{H z}\right)$ & $B_{k z}(k H z)$ \\
\hline $\begin{array}{c}\text { BS } 1 \text { (AIRTEL- } \\
\text { STADIUM) }\end{array}$ & -64.00 & 1.58 & 6.07 \\
\hline BS 2 (MTN-CLINIC) & -62.00 & 0.92 & 10.45 \\
\hline BS 3 (MTN-STADIUM) & -64.00 & 1.63 & 5.87 \\
\hline BS 4 (GLO-LAW) & -74.00 & 1.04 & 9.26 \\
\hline BS 5 (ETISALAT-LAW) & -79.00 & 1.22 & 7.84 \\
\hline
\end{tabular}

Table 4: Network performance of all base stations with respect to mobile user 4 (ETISALAT SIM)

\begin{tabular}{lccc}
\hline $\begin{array}{l}\text { MOBILE STATION 4 } \\
\text { (ETISALAT) }\end{array}$ & $P_{k z}(\mathrm{dBm})$ & $\omega_{k z}\left(\frac{\left[\frac{b i t}{\mathrm{~Hz}}\right]}{\mathrm{Hz}}\right)$ & $B_{k z}(\mathrm{KHz})$ \\
\hline BS 1 (AIRTEL-STADIUM) & -84.00 & 3.15 & 3.05 \\
BS 2 (MTN-CLINIC) & -64.00 & 2.97 & 3.23 \\
BS 3 (MTN-STADIUM) & -77.00 & 0.22 & 42.91 \\
BS 4 (GLO-LAW) & -90.00 & 2.70 & 3.56 \\
BS 5 (ETISALAT-LAW) & -84.00 & 2.75 & 3.49 \\
\hline
\end{tabular}

In Table 3, GLO SIM card for Mobile Station 3 was used, the received signal strength at AIRTEL base station improved from $-72 \mathrm{dBm}$ to $-64 \mathrm{dBm}$ which is a good signal strength as explained in step 2 of Section 4.0. The information transmitted over the allocated bandwidth of $6.07 \mathrm{KHz}$ was $1.58([\mathrm{bit} / \mathrm{s}] / \mathrm{Hz})$ and this is approximately the same as in Table 2 , which was the highest value on this table, while this was followed by MTN base station at Stadium with spectral link efficiency of 1.53 ([bit/s]/Hz).

While using ETISALAT SIM card for Mobile Station 4, the received signal strength at AIRTEL base station dropped from $-72 \mathrm{dBm}$ to $-84 \mathrm{dBm}$ as shown in Table 4. The information transmitted over the allocated bandwidth of $3.05 \mathrm{kHz}$ was 3.15 ([bit/s]/Hz) which is the highest spectral link efficiency value on this table, while this was followed by MTN base station at the University Health Centre (Clinic) with spectral link efficiency of 2.97 ([bit/s]/Hz).

When these four tables were compared based on Hartley's law that the maximum data rate of a physical communication link is proportional to its bandwidth in $\mathrm{Hz}$. Hence the maximum spectral link efficiency is proportional to the bandwidth used for the transmission of the data. Therefore the maximum spectral link efficiency that has been highlighted in different colors in the tables one to four are listed and arranged against the respective base station, and the first two base stations with the highest spectral link efficiency value were selected for the collocation arrangement with three operators on a telecommunication mast. 
Table 5: Network performance of the highlighted Base Stations

\begin{tabular}{lccc}
\hline BASE STATION SITES & $P_{k z}(\mathrm{dBm})$ & $\omega_{k z}\left[\frac{\mathrm{bit}}{\mathrm{s}}\right]$ & \\
BS 4 (GLO-LAW) & -87.00 & 3.17 & $B_{k z}(\mathrm{kHz})$ \\
BS 5 (ETISAT-LAW) & -95.00 & 2.98 & 3.02 \\
BS 1 (AIRTEL-STADIUM) & -72.00 & 1.55 & 6.17 \\
BS 3 (MTN-STADIUM) & -67.00 & 1.53 & 6.28 \\
BS 1 (AIRTEL-STADIUM) & -64.00 & 1.58 & 6.07 \\
BS 3 (MTN-STADIUM) & -64.00 & 1.63 & 5.87 \\
BS 1 (AIRTEL-STADIUM) & -84.00 & 3.15 & 3.05 \\
BS 2 (MTN-CLINIC) & -64.00 & 2.97 & 3.23 \\
\hline
\end{tabular}

From Table 5, the base station with the best spectral link efficiency was GLO base station with 3.17 ([bits/s]/Hz) and this was followed closely by AIRTEL base station with 3.15 ([bit/s]/Hz).

Hence the two base station sites selected based on the spectral link efficiency for the collocation arrangement was GLO base station at Faculty of Law and AIRTEL base station at Stadium. Now based on the proximity of the base stations to each other, GLO base station (host in collocation) which is very close to ETISALAT and MTN - CLINIC base stations will host both ETISALAT and MTN-CLINIC telecommunication operators. While AIRTEL-STADIUM will host MTNSTADIUM operator on its mast, meanwhile there is a vacant space on the AIRTEL mast which could accommodate a new entry.

Tables 1 through 4 displays the network performance of each of the base stations based on the spectral link efficiency of each when they are all transmitting at the same bit rate. Base stations 1, 3 and 4 have highest spectral link efficiency for mobile users (operators) one, which means that the allocated bandwidth which was proportional to the data rate was effectively utilized, while base station 2 has the least spectral link efficiency making use of large bandwidth at the same bit rate, hence at this point the bandwidth was underutilized. At mobile users 2 and 3, base station 5 and base station 1 are at the highest spectral link efficiency, while the spectral link efficiency of base station 5 dropped. There was an increase in that of base station 1, which means all along, base station 1 maintained the best spectral link efficiency on all the networks, thus it was selected to be a host in the collocation arrangement. Base station 4 maintained an average value which later shoot up steadily at mobile user 4 , and base station 2 which was low at mobile users 1, 2, and 3 suddenly shoots up at mobile user 4 as well, but based on the performance from mobile user 1 to mobile user 4, base station 4 was considered to be higher in spectral link efficiency value when compared with base stations 2 and 3 .

Considering Also from Tables 1 through 4, even though base station 2 has the best received power on the mobile users, it does not make the effective use of the power in transmitting the bit rate required, but base stations 1 and 4 made the effective use of the power in transmitting the bit rate required.

\section{CONCLUSION}

The approach used in this paper for the selection of the BTS for collocation sites reflect the practical deployment of the developed algorithm. It also demonstrates how the use of the spectral link efficiency as a determinant factor in the determination of collocation sites was carried out. The guiding principle emanates from the fact that where a BTS with highest spectral link efficiency with respect to all the operating networks on the same coverage area is been selected to host the collocation arrangement. The collocation scheme can only host any other two operators at maximum plus the host operators based on the $120^{\circ}$ aperture standard.

In this paper, the spectral link efficiency of each of the base station with respect to all the operating networks on the campus has been examined and the base stations with the highest spectral link efficiency had been selected to host the collocation arrangement because they have used the smallest allocated bandwidth to transmit the same information at the same rate and this justifies the effective use of the bandwidth, while other base stations have underutilized the bandwidth even at the same bit rate. Three operators at maximum were accommodated on each of the host base stations, and base station 1 and base station 4 will be host in the collocation arrangement, because they have efficiently utilized the allocated bandwidth, hence they have the highest spectral link efficiency.

\section{REFERENCES}

[1] Emeka Onuzuruike, Telecom Infrastructure Sharing as a Strategy for Cost Optimization and Revenue Generation : A Case Study of MTN Nigeria/Zain Nigeria Collocation, Blekinge Institute of Technology, 2008.

[2] PCIA Federal Siting Legislation Guidance, Wireless Facilities Deployment: Federal Regulation in the Middle Class Tax Relief and Job Creation Act of 2012, PCIA, 2012. 
[3] Emma Okonji, “Tackling Poor Telecoms Service with Co-location", www.thisdaylive.com/articles/tackling-poortelecoms-service-with-co-location, 06/12/2012, Accessed on November 6, 2013

[4] Kunle Azeez, "Improving Telecoms via Co-Location", 18/07/13, $\quad$ www.nationalmirroronline.net Lnew/improving-telecoms-via-co-location, Accessed on October 30, 2013.

[5] Singh R.K., "Assessment of Electromagnetic Radiation from Base Station Antenna", Indian Journal of Radio and Space Physics, vol. 41, 2012, pp 557-565.

[6] Telecommunications - Nigeria, "NCC guidelines promote efficiency savings through infrastructure sharing", 28/11/2012, www.internnationallawoffice.com/news, Accessed on October 10, 2013.

[7] Guideline on Collocation and Infrastructure Sharing, Nigerian Communications Commission.

[8] O.M. Sadiq, A.O. Oyelade and C.A.S Ukachukwu, "10 Years of Telecommunication Infrastructure Development In Nigeria", International Conference on Innovations in Engineering and Technology, Department of Civil \& Environmental Engineering, Faculty of Engineering, university of Lagos, Lagos Nigeria, August 8-10, 2011, pp.67-71.

[9] H.R. Andersen, J.P. McGeehan, “Optimizing Microcell Base Station Locations Using Simulated Annealing Techniques", Centers for Communications Research University of Bristol.
[10] Kozlowska E., "Basic Principle of Fuzzy Logic", 01/08/2012 http://access.fel.cvut.cz/rservice.php, Accessed November 24, 2013

[11] Emmanuel Pollakis, Renato L. G. Cavalcante and SławomirSta'nczak, "Base station selection for energy efficient network operation with the majorization-minimization algorithm", Fraunhofer Institute for Telecommunications, Heinrich Hertz Institute, June 27, 2012, vol 5, 2012.

[12] ITU Out Door Propagation Model, Propagation Data and Prediction Methods For The Planning Of Short Range Outdoor Radio Communication Systems And Radio Local Area Networks In The Frequency Range $300 \mathrm{MHZ}$ to $100 \mathrm{MHZ}$, R-REC-P.1411-1-200102, ITU, 1999-2001

[13] Bhunia C.T., "Technology Network and Internet", New Age International, 2006.

[14] Niraf Trivedi, TEMS Parameters (Speech), ADA Cellworks, Ahmedabad.

[15] $3^{\text {rd }}$ Generation Partnership Project, Technical Specification Group GSM/EDGE, Radio Access Network, Radio Transmission and Reception, 3GPP TS 05.05 v8.11.0 (2001-08), 3GPP, Release 1999,chapter 4

[16] Electric and Magnetics fields and Health, http://www.emfs.info/limits/limitsorganisations/icnirp, Accessed April 30, 2011 